\title{
A Three-Phase Sensitivity-Based Approach for Smooth Line- Switching in Islanded Microgrids
}

This paper was downloaded from TechRxiv (https://www.techrxiv.org).

\section{LICENSE}

CC BY 4.0

SUBMISSION DATE / POSTED DATE

26-06-2021 / 19-01-2022

\section{CITATION}

Pompodakis, Evangelos; Kryonidis, Georgios C.; Alexiadis, Minas (2021): A Three-Phase Sensitivity-Based Approach for Smooth Line-Switching in Islanded Microgrids. TechRxiv. Preprint. https://doi.org/10.36227/techrxiv.14852568.v2

$\mathrm{DOI}$

10.36227/techrxiv.14852568.v2 


\title{
A Three-Phase Sensitivity-Based Approach for Smooth Line-Switching in Islanded Microgrids
}

\author{
Evangelos E. Pompodakis, Georgios C. Kryonidis, Emmanuel Karapidakis, and Minas C. Alexiadis
}

\begin{abstract}
This paper deals with a new line-switching method that facilitates the network reconfiguration of islanded microgrids. Its distinct features include the ability to handle network asymmetries and the minimization of the line current during the switching action. This is attained by developing a three-phase sensitivity-based method to determine the operating set-points of the distributed generators (DGs) that minimize the current of the candidate line participating in the switching action. These set-points correspond to the positive-sequence powers as well as the negative- and zero-sequence currents of all DGs. Furthermore, the network constraints such as voltage limits and power limits of DGs are always satisfied. Simulations are performed in a balanced 33-bus islanded network as well as in the unbalanced IEEE 8500-node network to evaluate the performance of the proposed method.
\end{abstract}

Index Terms - Droop control, islanded microgrids, lineswitching, sensitivities, network reconfiguration.

\section{NOTATIONS}

Every variable with an arrow e.g $\vec{X}$ is a complex number. Every variable without an arrow e. $\mathrm{X}$ is an absolute number. The bold variables are vectors or matrices.

$|\cdot|$ denotes absolute value real $(\vec{X})$ denotes the real part of $\vec{X}$ imag $(\vec{X})$ denotes the imaginary part of $\vec{X}$ $\max (\boldsymbol{X})$ denotes the maximum absolute value of vector $\boldsymbol{X}$ $\min (\boldsymbol{X})$ denotes the minimum absolute value of vector $\boldsymbol{X}$

\section{NOMENCLATURE}

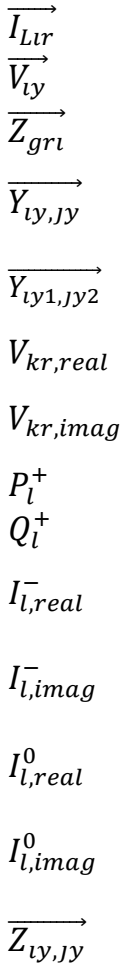

Load current of phase $r=\{a, b, c\}$ of bus $i$ Voltage of conductor $y=\{a, b, c, n, g\}$ of bus $i$ Grounding impedance of bus $i$

Self-admittance of conductor $y=\{a, b, c, n, g\}$ of the line between buses $i$ and $j$

Mutual-admittance of conductors $(y 1, y 2)=\{a$, $b, c, n, g\}$ of the line between buses $i$ and $j$

Real voltage component of phase $r$ of bus $k$ Imaginary voltage component of phase $r$ of bus $k$

Positive-sequence active power of DG $l$

Positive-sequence reactive power of DG $l$

Real component of negative-sequence current of DG $l$

Imaginary component of negative-sequence current of DG $l$

Real component of zero-sequence current of DG $l$

Imaginary component of zero-sequence current of DG $l$

Self-impedance of conductor $y$ of the line between buses $i$ and $j$ $\overrightarrow{Z_{\imath y 1, j y 2}}$

$\overline{V_{z r}}$

$V_{z}^{+}$

$\alpha$

$d p$

$d q$

$d i_{-} \quad$ Variation step of negative-sequence current

$d i_{0} \quad$ Variation step of zero-sequence current

$P_{D G z}^{\max } \quad$ Maximum active power of DG $z$

$P_{D G z}^{\min } \quad$ Minimum active power of DG $z$

$Q_{D G z}^{\max } \quad$ Maximum reactive power of $\mathrm{DG} z$

$Q_{D G z}^{\min } \quad$ Minimum reactive power of DG $z$

$P_{D G Z}$

Active power of DG $z$ derived from the power flow analysis

Reactive power of DG $z$ derived from the power

$Q_{D G Z} \quad$ flow analysis

$P_{\text {slack }} \quad$ Active power of slack bus derived from the power flow analysis

Reactive power of slack bus derived from the

$Q_{\text {slack }} \quad$ power flow analysis

$V_{\text {upper }}$ Maximum allowable phase-to-neutral voltage of the network

Vlower Minimum allowable phase-to-neutral voltage of the network

nom Nominal phase-to-neutral voltage of the network

$P_{\text {max }}^{\max } \quad$ Maximum active power of DG that is assumed as slack bus

Minimum active power of DG that is assumed as slack bus

Maximum reactive power of DG that is assumed as slack bus

Minimum reactive power of DG that is assumed as slack bus

Reference positive-sequence voltage of droop equation of $\mathrm{DG} z$

$\overrightarrow{V_{z, r e f}^{+}}$

$f_{\text {ref }} \quad$ Reference frequency of droop equation of DG $z$

$K_{q z} \quad$ Reactive power droop gain of DG $z$

$K_{p z} \quad$ Active power droop gain of DG $z$

\section{INTRODUCTION}

$\mathrm{N}$ ETWORK reconfiguration techniques are applied to islanded microgrids (MGs) to improve their operational characteristics in terms of loss minimization [1],[2], voltage profile improvement [3], loadability maximization [4], reliability improvement [5] and other criteria [6]-[9]. The 
network reconfiguration implies the connection or disconnection of lines to change the topology of the network. However, if the current flowing through the line at the moment of executing the switching action is high, several power quality and reliability problems may occur in addition to the wear and tear cost of the switching devices [11]. This problem is more severe in islanded microgrids since they are characterized by low levels of inertia [11].

In conventional distribution networks, switches are usually manually controlled and the line-switchings are executed only a few times per day. However, with the evolution of smart grids and the advent of MGs, all manual switches are expected to be replaced by automatic remotely-controlled switches, which may operate even in an hourly basis [10]. The frequent line-switching results in several negative impacts on the network, especially when the current flowing through the switched line is high at the time instant of switching. More specifically, in cases where a switch interrupts a line with a high current, the following negative impacts can appear in the network [11]:

- large disturbances caused by the line-switching in low inertia islanded MGs can trigger outages, which can threaten the stability of the system.

- Simulation results presented in [11] indicate that the voltage transients caused during the line-switching can cause damages in sensitive loads. For instance, looking at Fig. 1b, overvoltages more than $35 \%$ appear in the examined islanded MG of Fig. 1a, if the current of the switched line (L5) is higher than $0.6 \mathrm{pu}$. This overvoltage is significantly higher than the maximum overvoltage limit expressed in the IEEE standards [12],[13].

- the wear and tear of the switching devices increases if frequent interruptions of high current occur.

Ideally, the current flowing through the switched line should be zero to avoid the aforementioned negative impacts. Distributed generators (DGs) provide fast and flexible active and reactive power control and could be utilized to nullify the current of switched lines during the switching action. However, smoothing the line-switchings during the reconfiguration of MGs is currently an unexplored topic. To the best of our knowledge, only one paper exists in the literature so far to deal with this issue. More specifically, the authors in [11] formulate a dynamic mixed-integer nonlinear programming (MINP) problem to find the optimal active and reactive power output of DGs (by regulating the droop curves of DGs in droop controlled islanded microgrids) so that the power flowing through the switched line is minimized. However, in this study, the authors assume a balanced MG, which is not realistic since islanded MGs are generally highly unbalanced multigrounded 4-wire low voltage (LV) or medium voltage (MV) networks. Furthermore, the computation burden of MINP is too high, which makes the method dysfunctional, especially in large unbalanced networks.

This paper attempts to fill this gap by proposing a threephase sensitivity-based current minimization (SBCM) approach for smoothing the switching of the lines. The proposed method determines the positive-sequence output power as well as the negative- and zero-sequence currents of all DGs so that the magnitude of the currents flowing through each phase and neutral (if exists) of the switched line is minimum. The network constraints such as voltage limits and power limits of DGs are always satisfied. The proposed SBCM approach has the following distinct characteristics:

- High accuracy. The proposed method is characterized by high levels of accuracy since it considers the network unbalances as well as the effect of neutral and grounding in the power flow results.

- Reduced implementation complexity. The proposed method combines the proposed SBCM approach with the power flow algorithm, thus facilitating its implementation under realfield conditions.

Reduced computational complexity. The proposed algorithm presents very low computation time since it avoids the usage of optimization algorithms. More specifically, the optimal parameters of DGs are computed by executing only the power flow and the SBCM algorithm. The implicit Z-Bus power flow algorithm is applied in this paper, which is a robust and fast power flow solver. The proposed SBCM algorithm is executed after the execution of power flow and it has very low computation time. The computation time is a very important factor in such applications since the proposed algorithm must be executed directly before the switching action, in real-time, to minimize the switched power.

The rest of the paper is structured, as follows: Section II describes shortly the implicit Z-Bus method in multi-grounded distribution networks. Section III describes the proposed SBCM approach. Section IV includes simulation results in a balanced 33-bus islanded microgrid and the unbalanced IEEE 8500 -node network. Finally, Section V concludes the paper.
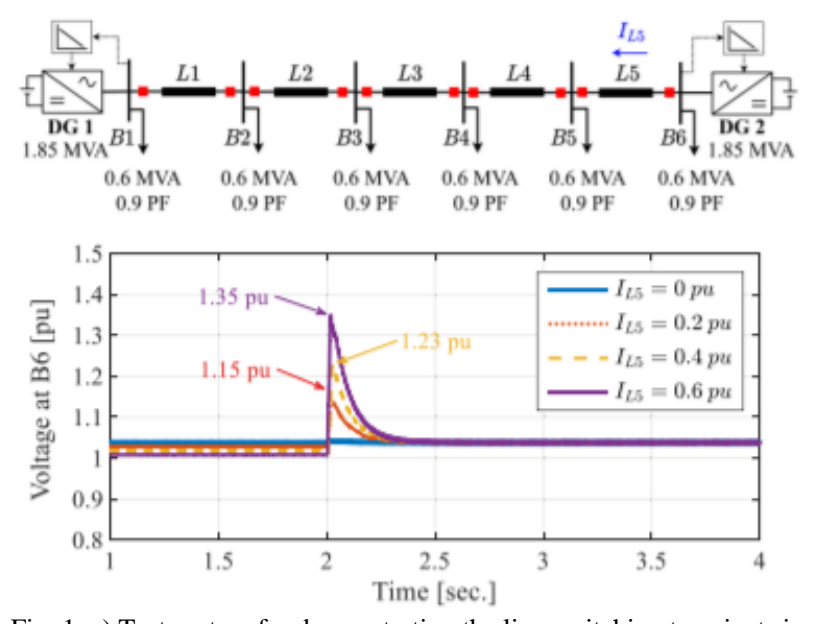

Fig. 1. a) Test system for demonstrating the line-switching transients in islanded MGs, and b) Voltage transients due to interrupting different linecurrent levels in the examined MG [11].

\section{MATHEMATICAL FORMULATION OF IMPLICIT Z-BUS POWER FLOW ALGORITHM}

The full configuration of an unbalanced MG is presented in Fig. 2. The introduction of network unbalances as well as the detailed modeling of the neutral conductor and the grounding system increases the power flow calculation accuracy of the 4wire multi-grounded islanded MGs, as demonstrated in [15] and [16]. The slack node of Fig. 2 corresponds to a DG with voltage balancing capability (generates balanced phase-toneutral voltage). Contrary to the use of a slack bus with fixed voltage in conventional power flow algorithms, the voltage of the slack bus is adjusted in our application to mitigate the 
overvoltages (or undervoltages) of the network. More details regarding the slack bus are presented in the next sections.

Let us define the current and voltage vectors of node $i$, as follows:

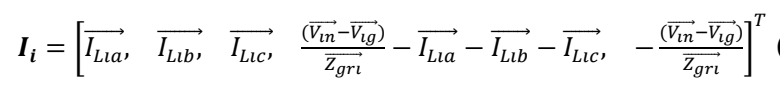

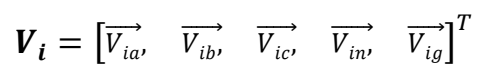

where $\overrightarrow{I_{L l r}}$ and $\overrightarrow{V_{l y}}$ denote the load current and voltage (in complex form) of node $i$ at phase $r=\{a, b, c\}$ and conductor $y=\{a, b, c, n, g\}$, respectively, as shown in Fig. 2 .

For a network with $m$ nodes, the current vectors can be expressed as a function of the voltage vectors as follows:

$$
\left[\begin{array}{c}
I_{0} \\
I_{1} \\
\vdots \\
I_{m}
\end{array}\right]=\left[\begin{array}{cccc}
Y_{00} & Y_{01} & \cdots & Y_{0 m} \\
Y_{10} & Y_{11} & \cdots & Y_{1 m} \\
\vdots & \vdots & \ddots & \vdots \\
Y_{m 0} & Y_{m 1} & \cdots & Y_{m m}
\end{array}\right]\left[\begin{array}{c}
V_{0} \\
V_{1} \\
\vdots \\
V_{m}
\end{array}\right]
$$

where $\boldsymbol{V}_{\mathbf{0}}$ coresponds to the slack node.

In (3), each non-diagonal element of the admittance matrix, e.g., $\boldsymbol{Y}_{i j}$ for $i \neq j$, is defined by (4), which includes the self- and mutual admittances of each line sector between the nodes $i$ and $j$. The diagonal elements of (3) are defined as: $\boldsymbol{Y}_{\boldsymbol{i} \boldsymbol{i}}=$ $-\sum_{k=0, k \neq i}^{m}\left(\boldsymbol{Y}_{\boldsymbol{i k}}\right)$

$$
\boldsymbol{Y}_{i j}=\left[\begin{array}{lllll}
\overrightarrow{Y_{i a, j a}} & \overrightarrow{Y_{i a, j b}} & \overrightarrow{Y_{i a, j c}} & \overrightarrow{Y_{i a, j n}} & \overrightarrow{Y_{i a, j g}} \\
\overrightarrow{Y_{i c, j a}} & \overrightarrow{Y_{i b, j b}} & \overrightarrow{Y_{i b, j c}} & \overrightarrow{Y_{i b, j b}} & \overrightarrow{Y_{i b, j g}} \\
\overrightarrow{Y_{i c, j c}} & \overrightarrow{Y_{i c, j n}} & \overrightarrow{Y_{i c, j g}} \\
\overrightarrow{Y_{i g, j a}} & \overrightarrow{Y_{i g, j b}} & \overrightarrow{Y_{i n, j c}} & \overrightarrow{Y_{i n, j n}} & \overrightarrow{Y_{i n, j g}} \\
\overrightarrow{Y_{i g, j n}} & \overrightarrow{Y_{i g, j g}}
\end{array}\right]
$$

Subsequently, for the power flow solution, we remove the first five rows of (3) that correspond to the slack node, and (5) is obtained.

$$
\left[\begin{array}{c}
I_{1} \\
\vdots \\
I_{m}
\end{array}\right]=\left[\begin{array}{cccc}
Y_{10} & Y_{11} & \cdots & Y_{1 m} \\
\vdots & \vdots & \ddots & \vdots \\
Y_{m 0} & Y_{m 1} & \cdots & Y_{m m}
\end{array}\right]\left[\begin{array}{c}
V_{0} \\
V_{1} \\
\vdots \\
V_{m}
\end{array}\right]
$$

Then, by transferring all the voltage variables of the lefthand side of (5) to the right-hand side, (6) is derived, where $\boldsymbol{I}_{\text {new }}$ and $\boldsymbol{Y}_{\text {new }}$ are the modified current and admittance matrices.

$$
I_{\text {new }}=Y_{n e w} V
$$

As a last step, we define the final matrices $\boldsymbol{Y}_{\text {fin }}^{\prime}$ and $\boldsymbol{Y}_{\text {fin }}$. The first one consists of the first five columns of $\boldsymbol{Y}_{\text {new }}$, while the second one consists of the remaining columns so that $\boldsymbol{Y}_{\text {new }}$ $=\left[\begin{array}{ll}\boldsymbol{Y}_{\text {fin }}^{\prime} & \boldsymbol{Y}_{\boldsymbol{f i n}}\end{array}\right]$. Eq. (7) is then derived from (6) by subtracting the product $\boldsymbol{Y}^{\prime}{ }_{\text {in }} \cdot \boldsymbol{V}_{\mathbf{0}}$ from both equation sides.

$$
-\boldsymbol{Y}_{\text {fin }}^{\prime} \cdot\left[\begin{array}{l}
\overrightarrow{V_{0 a}} \\
\overrightarrow{V_{0 b}} \\
\overrightarrow{V_{0 c}}
\end{array}\right]+\boldsymbol{I}_{\text {new }}=-\boldsymbol{Y}_{\text {fin }}^{\prime} \cdot\left[\begin{array}{l}
\overrightarrow{V_{0 n}} \\
\overrightarrow{V_{0 g}}
\end{array}\right]\left[\begin{array}{l}
\overrightarrow{V_{0 c}} \\
\overrightarrow{V_{0 n}} \\
\overrightarrow{V_{0 g}}
\end{array}\right]+\boldsymbol{Y}_{\text {new }} \cdot \boldsymbol{V}
$$

Using (7), we finally derive (8), which is iteratively solved until a certain preset tolerance is reached. In (8), $k$ denotes the iteration number, and the vector $\boldsymbol{V}_{\text {fin }}$ contains the voltages of all nodes except the slack.

$$
\boldsymbol{Y}_{\boldsymbol{f i n}}{ }^{-1} \cdot\left[-\boldsymbol{Y}_{\boldsymbol{f i n}}^{\prime} \cdot \boldsymbol{V}_{\mathbf{0}}+\boldsymbol{I}_{\boldsymbol{n e w}}\right]^{k}=\boldsymbol{V}_{\boldsymbol{f i n}}{ }^{k+1}
$$

The vector $\boldsymbol{V}_{\mathbf{0}}$ represents the voltage of slack node and it is balanced. In our algrithm, the magnitude of the slack node will be varied based on the power flow results so that the voltage contraints of all nodes of the network are satisfied, as it will be explained in the next sections.

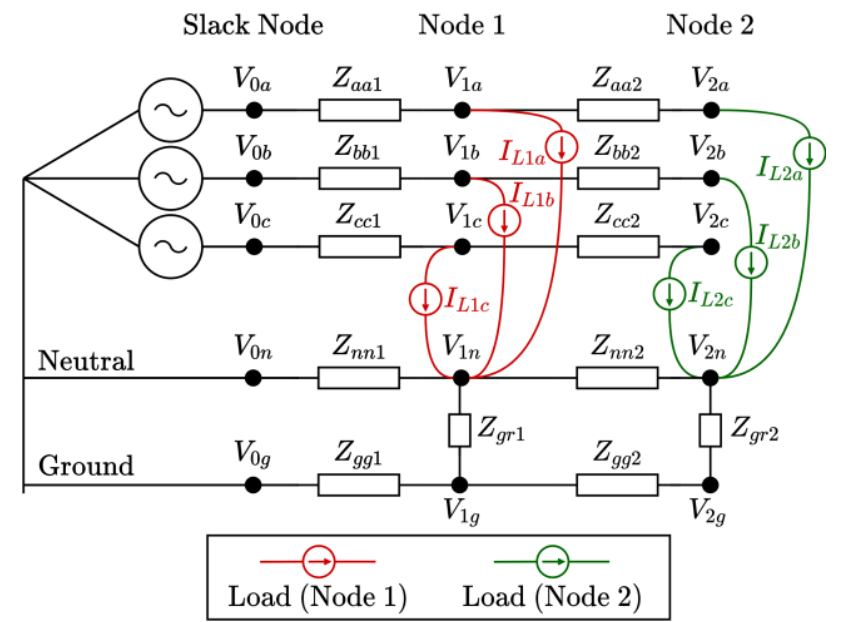

Fig. 2. Equivalent circuit of a microgrid consisting of a DG with balanced voltage (it is assumed as slack node) and two load nodes. The loads are connected between the phases and the multi-grounded neutral conductor.

\section{Proposed Sensitivity-BASEd Method}

The section is divided in three sub-sections. In the first, the proposed SBCM approach is described. In the second, the mathematical derivation of sensitivity parameters is presented, while in the third, the constraints of the SBCM method are quoted.

\section{A) Description of the proposed method}

Let us assume that we have a three-phase 4-wire network with $m$ buses that hosts a $d$ number of DGs. Let us further assume that the network is to be reconfigured by connecting the buses $k$ and $p$ through a 4 -wire line. To achieve a smooth switching action, the current through the new line at the time instant of the connection should be as low as possible, or equivalently, the voltage difference between the buses $k$ and $p$ should be minimum. Therefore, the objective of the SBCM method is to equalize the voltage of buses $k$ and $p$.

The variation of the real and imaginary components of the voltage of buses $k$ and $p$ are given in (9), as a function of the variation of the positive-sequence powers as well as the real and imaginary components of negative- and zero-sequence currents of DGs. It is pointed out, that in this paper, we use as control variables the positive-sequence active and reactive power as well as the real and imaginary components of negative- and zero-sequence currents of DGs. It is because most of the existing control schemes of IBDGs operate in $d q 0$ coordinates, regulating independently, the sequence components of current (see section IV). Nevertheless, if an IBDG is controlled in $a b c$ coordinates, the real and imaginary components of phase currents could be used, instead, as control variables. 


$$
\left[\begin{array}{c}
d V_{\text {ka,real }} \\
d V_{\text {kb,real }} \\
d V_{\text {kc,real }} \\
d V_{\text {ka,imag }} \\
d V_{\text {kb,imag }} \\
d V_{\text {kc,imag }} \\
d V_{\text {pa,real }} \\
d V_{\text {pb,real }} \\
d V_{\text {pc,real }} \\
d V_{\text {pa,imag }} \\
d V_{p b, \text { imag }} \\
d V_{p c, \text { imag }}
\end{array}\right]=\text { Sens } \cdot\left[\begin{array}{c}
d P_{1}^{+} \\
d Q_{1}^{+} \\
d I_{1, \text { real }}^{-} \\
d I_{1, \text { imag }}^{-} \\
d I_{1, \text { real }}^{0} \\
d I_{1, \text { imag }}^{0} \\
\vdots \\
d P_{d}^{+} \\
d Q_{d}^{+} \\
d I_{d, \text { real }}^{-} \\
d I_{d, \text { imag }}^{-} \\
d I_{d, \text { real }}^{0} \\
d I_{d, \text { imag }}^{0}
\end{array}\right]
$$

In (9), the sensitivity matrix Sens (eq. (10)) is provided at the end of the paper. $d P_{l}^{+}, d Q_{l}^{+}, d I_{l, \text { real }}^{-}, d I_{l, \text { imag }}^{-}, d I_{l, \text { real }}^{0}, d I_{l, \text { imag }}^{0}$ is the variation of the positive-sequence active power, positivesequence reactive power, real component of negativesequence current, imaginary component of negative-sequence current, real component of zero-sequence current, imaginary component of zero-sequence current of DG $l$, respectively. $d V_{k r, \text { real }}, d V_{k r, \text { imag }}, d V_{p r, \text { real }}, d V_{p r, \text { imag }}$ is the variation of the real and imaginary components of the voltage of phase $r$ of buses $k$ and $p$, respectively.

Eq. (11) is easily obtained from (9) by subtracting the last six rows from the first six rows of Sens matrix. Sens $\boldsymbol{s o d}_{\text {mod }}$ is the modified Sens matrix and it has dimension $6 \times 6 \cdot d$, where $d$ is the number of DGs.

$$
\left[\begin{array}{c}
d V_{\text {ka,real }}-d V_{\text {pa,real }} \\
d V_{k b, \text { real }}-d V_{\text {pb,real }} \\
d V_{\text {kc,real }}-d V_{\text {pc,real }} \\
d V_{\text {ka,imag }}-d V_{\text {pa,imag }} \\
d V_{\text {kb,imag }}-d V_{\text {pb,imag }} \\
d V_{k c, \text { imag }}-d V_{p c, \text { imag }}
\end{array}\right]=\text { Sens }_{\text {mod }} \cdot\left[\begin{array}{c}
d P_{1}^{+} \\
d Q_{1}^{+} \\
d I_{1, \text { real }}^{-} \\
d I_{1, \text { imag }} \\
d I_{1, \text { real }}^{0} \\
d I_{1, \text { imag }}^{0} \\
\vdots \\
d P_{d}^{+} \\
d Q_{d}^{+} \\
d I_{d, \text { real }}^{-} \\
d I_{d, \text { imag }}^{-} \\
d I_{d, \text { real }}^{0} \\
d I_{d, \text { imag }}^{0}
\end{array}\right]
$$

The objective function that needs to be minimized is given by (12).

$$
\begin{gathered}
\text { Cost }=\left|\left(V_{k a, \text { real }}-V_{p a, \text { real }}\right)+\left(d V_{\text {ka,real }}-d V_{\text {pa,real }}\right)\right| \\
+\left|\left(V_{k b, \text { real }}-V_{p b, \text { real }}\right)+\left(d V_{k b, \text { real }}-d V_{p b, \text { real }}\right)\right| \\
+\left|\left(V_{k c, \text { real }}-V_{p c, \text { real }}\right)+\left(d V_{k c, \text { real }}-d V_{p c, \text { real }}\right)\right| \\
+\left|\left(V_{k a, \text { imag }}-V_{p a, \text { imag }}\right)+\left(d V_{k a, \text { imag }}-d V_{\text {pa,imag }}\right)\right| \\
+\left|\left(V_{k b, \text { imag }}-V_{p b, \text { imag }}\right)+\left(d V_{k b, \text { imag }}-d V_{p b, \text { imag }}\right)\right| \\
+\left|\left(V_{k c, \text { imag }}-V_{p c, \text { imag }}\right)+\left(d V_{k c, \text { imag }}-d V_{p c, \text { imag }}\right)\right|
\end{gathered}
$$

In (12), $V_{k r, \text { real }}, V_{k r, \text { imag }}, V_{p r, \text { real }}, V_{p r, i m a g}$ of phase $r$ are calculated from the power flow. The difference of differentials $\left(d V_{k r, \text { real }}-d V_{p r, \text { real }}\right)$ and $\left(d V_{k r, \text { imag }}-d V_{p r, i m a g}\right)$ is calculated from (11), using the following procedure.

We define four small steps e.g., $d p, d q, d i_{-}, d i_{0}$ for the active power, reactive power, negative- and zero-sequence currents, respectively. The value of each step is empirically selected. In our simulations, we selected them as a small fraction (around 5\%) of the maximum powers or currents of DGs. After the convergence of the power flow, we search for the action that implies the lowest cost in (12), considering only one action $\left(+d p\right.$ or $+d q$ or $+d i_{-}$or $+d i_{0}$ or $-d p$ or $-d q$ or $-d i_{-}$ or $-d i_{0}$ ) from only one DG at each time. For instance, let us ) assume that the action $+d p$ of the positive-sequence active power of DG $d$ (namely $d P_{d}^{+}=+d p$ in (11)) leads to the lowest value of Cost function in (12). Then, we increase $P_{d}^{+}$by $+d p$ and the power flow is executed again. This process is repeated until the phase and neutral currents of the switched line are minimized. Based on our experience, ideally the steps $+d p,+d q,+d i_{-},+d i_{0},-d p,-d q,-d i_{-},-d i_{0}$ should be multiplied by the parameter rand, where rand is a uniformly distributed random number between $[0,1]$. In this way, the proposed SBCM approach is not stuck in contrasting actions e.g., $+d p$ $\rightarrow-d p \rightarrow+d p \rightarrow-d p$ from the same DG.

\section{B) Derivation of Sensitivity Parameters}

In this sub-section, the elements of Sens matrix (see (10) at the end of the paper) are derived. More specifically, the sensitivity of the real and imaginary voltage components of bus $q=\{k, p\}$ from the control variables of DG $z=\{1, \ldots, d\}$ (e.g., $P_{z}^{+}, Q_{z}^{+}, I_{z, \text { real }}^{-}, I_{z, \text { imag }}^{-}, I_{z, \text { real }}^{0}, I_{z, \text { imag }}^{0}$ ) is defined.

Firstly, it is necessary to define the impedance matrix of the network $\left(\boldsymbol{Z}_{\text {matr }}\right)$, as shown in (13).

$$
Z_{m a t r}=\left[\begin{array}{ccc}
Z_{11} & \cdots & Z_{1 m} \\
\vdots & \ddots & \vdots \\
Z_{m 1} & \cdots & Z_{m m}
\end{array}\right]
$$

where the element $\boldsymbol{Z}_{\boldsymbol{i} \boldsymbol{j}}$ of $\boldsymbol{Z}_{\text {matr }}$ is denoted in (14). In fact, $Z_{\text {matr }}$ is equal to $\boldsymbol{Y}_{\boldsymbol{f i n}}{ }^{-1}$ in (8).

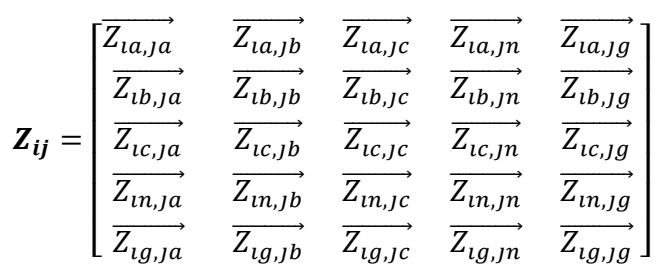

1. Derivation of $\frac{d V_{\text {qr,real }}}{d P_{z}^{+}}$and $\frac{d V_{\text {qr,imag }}}{d P_{z}^{+}}$for phase $r$

A perturbation of the positive-sequence active power of DG $z$ equal to $d p$ will cause a perturbation of the three phase currents of DG $z$, as follows:

$$
\left[\begin{array}{c}
\overrightarrow{d I_{z a}} \\
\overrightarrow{d I_{z b}} \\
\overrightarrow{d I_{z c}}
\end{array}\right]=\left[\begin{array}{c}
1 \\
\alpha^{2} \\
\alpha
\end{array}\right] \cdot \frac{d p}{{\overrightarrow{V_{z}^{+}}}^{*}}
$$

where ${\overrightarrow{V_{z}^{+}}}^{*}$ is the complex conjugate of the positive-sequence voltage of bus $z$.

The perturbed currents of (15), will cause a perturbation of the real and imaginary components of voltage of bus $q$, as explained in (16), (17), respectively. 


$$
\begin{array}{r}
d V_{q r, \text { real }}=\operatorname{real}\left(\left[\begin{array}{lll}
\overrightarrow{Z_{q r, z a}} & \overrightarrow{Z_{q r, z b}} & \overrightarrow{Z_{q r, z c}}
\end{array}\right] \cdot\left[\begin{array}{l}
\overrightarrow{d_{z a}} \\
\overrightarrow{d I_{z b}}
\end{array}\right]\right) \\
d V_{q r, \text { imag }}=\operatorname{imag}\left(\left[\begin{array}{lll}
\overrightarrow{Z_{q r, z a}} & \overrightarrow{Z_{q r, z b}} & \overrightarrow{Z_{q r, z c}}
\end{array}\right] \cdot\left[\begin{array}{l}
\overrightarrow{d I_{z a}} \\
\overrightarrow{d I_{z b}}
\end{array}\right)\right)
\end{array}
$$

The sensitivity parameters are calculated by dividing (16) and (17) with $d p$ (e.g $\frac{d V_{q r, \text { real }}}{d P_{\mathrm{Z}}^{+}}=\frac{d V_{q r, \text { real }}}{d p}$ ).

2. Derivation of $\frac{d V_{q r, \text { real }}}{d Q_{Z}^{+}}$and $\frac{d V_{q r, \text { imag }}}{d Q_{Z}^{+}}$for phase $r$

A perturbation of the positive-sequence reactive power of DG $z$ equal to $d q$ will cause a perturbation of the three phase currents of DG $z$, as follows:

$$
\left[\begin{array}{l}
\overrightarrow{d I_{z a}} \\
\overrightarrow{d I_{z b}}
\end{array}\right]=\left[\begin{array}{c}
1 \\
\alpha^{2} \\
\alpha
\end{array}\right] \cdot \frac{-j \cdot d q}{{\overrightarrow{V_{z}^{+}}}^{*}}
$$

The perturbed currents of (18), will cause a perturbation at the real and imaginary components of bus $q$, as explained in (19), (20), respectively.

$$
\begin{array}{r}
d V_{q r, \text { real }}=\operatorname{real}\left(\left[\begin{array}{lll}
\overrightarrow{Z_{q r, z a}} & \overrightarrow{Z_{q r, z b}} & \overrightarrow{Z_{q r, z c}}
\end{array}\right] \cdot\left[\begin{array}{l}
\overrightarrow{d I_{z a}} \\
\frac{\overrightarrow{d I_{z b}}}{d I_{z c}}
\end{array}\right]\right) \\
d V_{q r, \text { imag }}=\operatorname{imag}\left(\left[\begin{array}{lll}
\overrightarrow{Z_{q r, z a}} & \overrightarrow{Z_{q r, z b}} & \overrightarrow{Z_{q r, z c}}
\end{array}\right] \cdot\left[\begin{array}{l}
\overrightarrow{d I_{z a}} \\
\frac{\overrightarrow{d I_{z b}}}{d I_{z c}}
\end{array}\right]\right)
\end{array}
$$

The sensitivity parameters are calculated by dividing (19) and (20) with $d q$.

3. Derivation of $\frac{d V_{\text {qr,real }}}{d I_{z, \text { real }}^{-}}$and $\frac{d V_{\text {qrimag }}}{d I_{z, \text { real }}^{-}}$for phase $r$

A perturbation of the real component of negative-sequence current of DG $z$ equal to $d i_{-}$will cause a perturbation of the three phase currents of DG $z$, as follows:

$$
\left[\begin{array}{l}
\overrightarrow{d I_{z a}} \\
\overrightarrow{d I_{z b}}
\end{array}\right]=\left[\begin{array}{c}
1 \\
\alpha \\
\alpha^{2}
\end{array}\right] \cdot d i_{-}
$$

The perturbed currents of (21), will cause a perturbation at the real and imaginary components of bus $q$, as follows:

$$
\begin{array}{r}
d V_{q r, r e a l}=\operatorname{real}\left(\left[\begin{array}{lll}
\overrightarrow{Z_{q r, z a}} & \overrightarrow{Z_{q r, z b}} & \overrightarrow{Z_{q r, z c}}
\end{array}\right] \cdot\left[\begin{array}{l}
\overrightarrow{d I_{z a}} \\
\overrightarrow{d I_{z b}}
\end{array}\right]\right) \\
d V_{q r, i m a g}=\operatorname{imag}\left(\left[\begin{array}{lll}
\overrightarrow{Z_{q r, z a}} & \overrightarrow{Z_{q r, z b}} & \overrightarrow{Z_{q r, z c}}
\end{array}\right] \cdot\left[\begin{array}{l}
\overrightarrow{d I_{z a}} \\
\overrightarrow{d I_{z b}} \\
\overrightarrow{d I_{z c}}
\end{array}\right]\right)
\end{array}
$$

The sensitivity parameters are calculated by dividing (22) and (23) with $d i_{-}$.

4. Derivation of $\frac{d V_{q r, r e a l}}{d I_{z, \text { imag }}^{-}}$and $\frac{d V_{\text {qr,imag }}}{d I_{z, \text { imag }}^{-}}$for $r=\{a, b, c\}$
A perturbation of the imaginary component of negativesequence current of DG $z$ equal to $d i_{-}$will cause a perturbation of the three phase currents of $\mathrm{DG} z$, as follows:

$$
\left[\begin{array}{l}
\overrightarrow{d I_{z a}} \\
\overrightarrow{d I_{z b}}
\end{array}\right]=\left[\begin{array}{c}
1 \\
\alpha \\
\alpha^{2}
\end{array}\right] \cdot j \cdot d i_{-}
$$

The perturbed currents of (24), will cause a perturbation at the real and imaginary components of bus $q$, as follows:

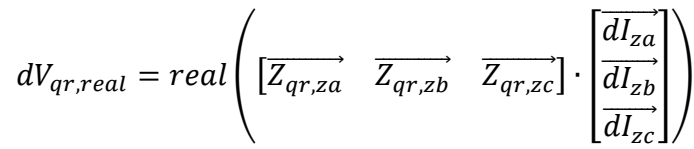

$$
\begin{aligned}
& d V_{q r, i m a g}=\operatorname{imag}\left(\left[\begin{array}{lll}
\overrightarrow{Z_{q r, z a}} & \overrightarrow{Z_{q r, z b}} & \overrightarrow{Z_{q r, z c}}
\end{array}\right] \cdot\left[\begin{array}{l}
\overrightarrow{d I_{z a}} \\
\overrightarrow{d I_{z b}} \\
\overrightarrow{d I_{z c}}
\end{array}\right]\right)
\end{aligned}
$$

The sensitivity parameters are calculated by dividing (25) and (26) with $d i_{-}$.

5. Derivation of $\frac{d V_{q r, \text { real }}}{d I_{z, \text { real }}^{0}}$ and $\frac{d V_{q r, \text { imag }}}{d I_{z, \text { real }}^{0}}$ for $r=\{a, b, c\}$

A perturbation of the real component of zero-sequence current of DG $z$ equal to $d i_{0}$ will cause a perturbation of the three phase currents of DG $z$, as follows:

$$
\left[\begin{array}{l}
\overrightarrow{d I_{z a}} \\
\overrightarrow{d I_{z b}} \\
\overrightarrow{d I_{z c}}
\end{array}\right]=\left[\begin{array}{c}
1 \\
1 \\
1 \\
-3
\end{array}\right] \cdot d i_{0}
$$

The perturbed currents of (27), will cause a perturbation at the real and imaginary components of bus $q$, as follows:

$$
\begin{aligned}
& \left.d V_{q r, \text { real }}=\operatorname{real}\left(\begin{array}{llll}
\overrightarrow{Z_{q r, z a}} & \overrightarrow{Z_{q r, z b}} & \overrightarrow{Z_{q r, z c}} & \overrightarrow{Z_{q r, z n}}
\end{array}\right] \cdot\left[\begin{array}{l}
\overrightarrow{\frac{d I_{z a}}{d I_{z b}}} \\
\frac{d I_{z c}}{d I_{z n}}
\end{array}\right]\right) \\
& d V_{q r, \text { imag }}=\operatorname{imag}\left(\left[\begin{array}{llll}
\overrightarrow{Z_{q r, z a}} & \overrightarrow{Z_{q r, z b}} & \overrightarrow{Z_{q r, z c}} & \overrightarrow{Z_{q r, z n}}
\end{array}\right] \cdot\left[\begin{array}{l}
\overrightarrow{\frac{d I_{z a}}{d I_{z b}}} \\
\overrightarrow{\frac{d I_{z c}}{\overrightarrow{d I}}}
\end{array}\right]\right)
\end{aligned}
$$

The sensitivity parameters are calculated by dividing (28) and (29) with $d i_{0}$.

6. Derivation of $\frac{d V_{q r, \text { real }}}{d I_{z, \text { imag }}^{0}}$ and $\frac{d V_{q r, i m a g}}{d I_{z, \text { imag }}^{0}}$ for $r=\{a, b, c\}$

A perturbation of the imaginary component of zerosequence current of DG $z$ equal to $d i_{0}$ will cause a perturbation of the three phase currents of DG $z$, as follows:

$$
\left[\begin{array}{l}
\overrightarrow{d I_{z a}} \\
\overrightarrow{d I_{z b}} \\
\overrightarrow{d I_{z c}}
\end{array}\right]=\left[\begin{array}{c}
1 \\
1 \\
1 \\
-3
\end{array}\right] \cdot j \cdot d i_{0}
$$

The perturbed currents of (30), will cause a perturbation at the real and imaginary components of bus $q$, as follows: 


$$
\begin{aligned}
d V_{q r, \text { real }}=\operatorname{real}\left(\left[\begin{array}{llll}
\overrightarrow{Z_{q r, z a}} & \overrightarrow{Z_{q r, z b}} & \overrightarrow{Z_{q r, z c}} & \overrightarrow{Z_{q r, z n}}
\end{array}\right] \cdot\left[\begin{array}{l}
\overrightarrow{d I_{z a}} \\
\overrightarrow{d I_{z b}} \\
\overrightarrow{d I_{z c}}
\end{array}\right]\right) \\
d V_{q r, \text { imag }}=\operatorname{imag}\left(\left[\begin{array}{llll}
\overrightarrow{Z_{q r, z a}} & \overrightarrow{Z_{q r, z b}} & \overrightarrow{Z_{q r, z c}} & \overrightarrow{Z_{q r, z n}}
\end{array}\right] \cdot\left[\begin{array}{l}
\overrightarrow{d I_{z a}} \\
\overrightarrow{d I_{z b}}
\end{array}\right]\right)
\end{aligned}
$$

The sensitivity parameters are calculated by dividing (31) and (32) with dio.

\section{C) Control Constraints}

The actions described in the previous sub-sections should be constrained based on the maximum active and reactive power of DGs as well as the voltage limits of the buses. For instance, assuming that DG $z$ has reached its maximum active power limit, the action $+d p$ for this DG is not a possible action. All constraints of the proposed SBCM approach are described below:

Constraint 1: + dp action for $P_{z}^{+}$of $D G z$

$$
\begin{gathered}
P_{D G z}+d p<P_{D G z}^{\max } \\
P_{\text {slack }}-d p>P_{\text {slack }}^{\min } \\
\max \left(\boldsymbol{V}_{p h-n}+\boldsymbol{Z}_{\boldsymbol{a b c}, \boldsymbol{a b c z}} \cdot\left[\begin{array}{c}
1 \\
\alpha^{2} \\
\alpha
\end{array}\right] \cdot \frac{d p}{{\overrightarrow{\overrightarrow{+}^{+}}}^{*}}\right)-\min \left(\boldsymbol{V}_{p h-n}+\boldsymbol{Z}_{\boldsymbol{a b c}, \boldsymbol{a b c z}}\right. \\
\left.\left[\begin{array}{c}
1 \\
\alpha^{2} \\
\alpha
\end{array}\right] \cdot \frac{d p}{\overrightarrow{V_{z}^{+}}}\right)<V_{\text {limit }}^{\text {upper }}-V_{\text {limit }}^{\text {lower }}
\end{gathered}
$$

Eq. (33) prevents the DG $z$ from exceeding its active power limit $\left(P_{D G z}^{\max }\right) . P_{D G z}$ is the output power of DG $z$ calculated in the last iteration of power flow (refer to section III.D).

Eq. (34) prevents the active power of slack bus (it is reminded that the slack bus corresponds to a DG with balanced phase-to-neutral voltage) from falling below its minimum power (usually 0). More specifically, if the power of DG $z$ increases by $d p$, inevitably the slack power will be reduced almost by $d p$. Since the slack bus of the power flow corresponds to a DG, the lower power limit of this DG $\left(P_{\text {slack }}^{\min }\right)$ should not be exceeded.

Finally, (35) ensures that a rise of positive-sequence active power of DG $z$ by $+d p$, will not cause a deviation between maximum and minimum voltage of the network higher than $V_{\text {limit }}^{\text {upper }}-V_{\text {limit }}^{\text {lower }}$, where $V_{\text {limit }}^{\text {upper }}$ and $V_{\text {limit }}^{\text {lower }}$ are the maximum and minimum allowable voltages e.g., $1.1 \cdot V_{\text {nom }}$ and $0.9 \cdot V_{\text {nom }}$, respectively [17], where $V_{\text {nom }}$ is the nominal voltage of the network. If (35) is satisfied, then by adjusting suitably the voltage of slack bus (see eq. (47)), all the voltages will remain between $V_{\text {limit }}^{\text {lower }}$ and $V_{\text {limit }}^{\text {upper }}$. It is pointed out that the vector $\boldsymbol{V}_{p h-n}$ in (35) expresses all phase-to-neutral voltages of the network ( $m$ is the number of buses) calculated at the last power flow iteration, as follows:

$$
\boldsymbol{V}_{p h-n}=\left[\begin{array}{c}
\overrightarrow{V_{1 a}}-\overrightarrow{V_{1 n}} \\
\overrightarrow{V_{1 b}}-\overrightarrow{V_{1 n}} \\
\overrightarrow{V_{1 c}}-\overrightarrow{V_{1 n}} \\
\vdots \\
\overrightarrow{V_{m a}}-\overrightarrow{V_{m n}} \\
\overrightarrow{V_{m b}}-\overrightarrow{V_{m n}} \\
\overrightarrow{V_{m c}}-\overrightarrow{V_{m n}}
\end{array}\right]
$$

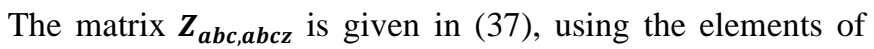
$Z_{\text {matr }}$ matrix of (13).

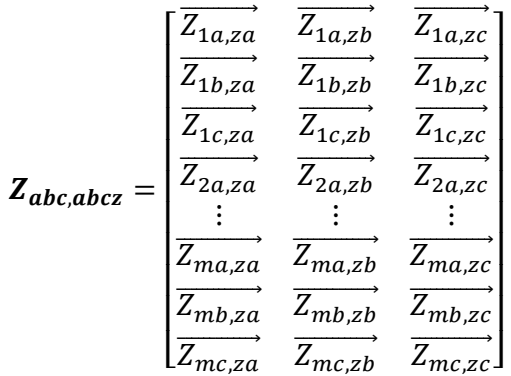

Constraint 2: $+d q$ action for $Q_{Z}^{+}$of $D G z$

$$
\begin{gathered}
Q_{D G z}+d q<Q_{D G z}^{\max } \\
Q_{\text {slack }}-d q>Q_{\text {slack }}^{\min } \\
\max \left(\boldsymbol{V}_{p h-n}+\boldsymbol{Z}_{\boldsymbol{a b c}, \boldsymbol{a b c z}} \cdot\left[\begin{array}{c}
1 \\
\alpha^{2} \\
\alpha
\end{array}\right] \cdot \frac{-j \cdot d q}{{\overrightarrow{V_{z}^{+}}}^{*}}\right)-\min \left(\boldsymbol{V}_{p h-n}+\boldsymbol{Z}_{\boldsymbol{a b c}, \boldsymbol{a b c z}}\right. \\
\left.\left[\begin{array}{c}
1 \\
\alpha^{2} \\
\alpha
\end{array}\right] \cdot \frac{-j \cdot d q}{{\overrightarrow{V_{z}^{+}}}^{*}}\right)<V_{\text {limit }}^{\text {upper }}-V_{\text {limit }}^{\text {lower }}
\end{gathered}
$$

Constraint 3: +di_action for $I_{z, \text { real }}^{-}$of $D G z$

$$
\begin{array}{r}
\max \left(\boldsymbol{V}_{p h-n}+\boldsymbol{Z}_{\boldsymbol{a b c}, \boldsymbol{a b c z}} \cdot\left[\begin{array}{c}
1 \\
\alpha \\
\alpha^{2}
\end{array}\right] \cdot d i_{-}\right)-\min \left(\boldsymbol{V}_{p h-n}+\boldsymbol{Z}_{\boldsymbol{a b c}, \boldsymbol{a b c z}} \cdot\left[\begin{array}{c}
1 \\
\alpha \\
\alpha^{2}
\end{array}\right]\right. \\
\left.d i_{-}\right)<V_{\text {limit }}^{\text {upper }}-V_{\text {limit }}^{\text {lower }}
\end{array}
$$

Equation (41) ensures that the variation of $I_{z, \text { real }}^{-}$by $+d i_{-}$ will not increase the deviation between maximum and minimum phase-to-neutral voltages beyond a limit. It is similar to (35) of constraint 1.

It is clarified that in this study, for simplicity, active and reactive power limits for the negative and zero-sequence components were not considered due to the very low negativeand zero-sequence powers compared with the positivesequence ones. Therefore, the active and reactive power limits of DGs are determined, exclusively, by the positive sequence component.

Constraint 4: + di_ action for $I_{z, \text { imag }}^{-}$of $D G z$

$$
\begin{gathered}
\max \left(\boldsymbol{V}_{p h-n}+\boldsymbol{Z}_{\boldsymbol{a b c} \boldsymbol{a} \boldsymbol{a b c z}} \cdot\left[\begin{array}{c}
1 \\
\alpha \\
\alpha^{2}
\end{array}\right] \cdot j \cdot d i_{-}\right)-\min \left(\boldsymbol{V}_{p h-n}+\boldsymbol{Z}_{\boldsymbol{a b c} \boldsymbol{a} \boldsymbol{a b c z}} \cdot\left[\begin{array}{c}
1 \\
\alpha \\
\alpha^{2}
\end{array}\right] .\right. \\
\left.j \cdot d i_{-}\right)<V_{\text {limit }}^{\text {upper }}-V_{\text {limit }}^{\text {lower }}
\end{gathered}
$$

Constraint 5: + di $i_{0}$ action for $I_{z, \text { real }}^{0}$ of $D G z$ 


$$
\begin{gathered}
\max \left(\boldsymbol{V}_{p h-n}+\left(\boldsymbol{Z}_{\boldsymbol{a b c} \boldsymbol{a} \boldsymbol{a b c n z}}-\boldsymbol{Z}_{\boldsymbol{n}, \boldsymbol{a b c n z}}\right) \cdot\left[\begin{array}{c}
1 \\
1 \\
1 \\
-3
\end{array}\right] \cdot d i_{0}\right)-\min \left(\boldsymbol{V}_{p h-n}+\right. \\
\left.\left(\boldsymbol{Z}_{\boldsymbol{a b c}, \boldsymbol{a b c n z}}-\boldsymbol{Z}_{\boldsymbol{n}, \boldsymbol{a b c n z}}\right) \cdot\left[\begin{array}{c}
1 \\
1 \\
1 \\
-3
\end{array}\right] \cdot d i_{0}\right)<V_{\text {limit }}^{\text {upper }}-V_{\text {limit }}^{\text {lower }}
\end{gathered}
$$

Constraint 5 ensures that the variation of $I_{z \text {,real }}^{0}$ by $+d i_{0}$ will not increase the deviation between maximum and minimum phase-to-neutral voltages beyond a limit. The main difference of constraint 5 against constraints 3 and 4 is that the zerosequence current returns through the neutral conductor, and therefore, the voltage of the neutral is varied, as well. The matrices $\boldsymbol{Z}_{\boldsymbol{a b c , \boldsymbol { a b c n z }},}, \boldsymbol{Z}_{\boldsymbol{n}, \boldsymbol{a b c n z}}$ are given by (44) and (45), respectively.

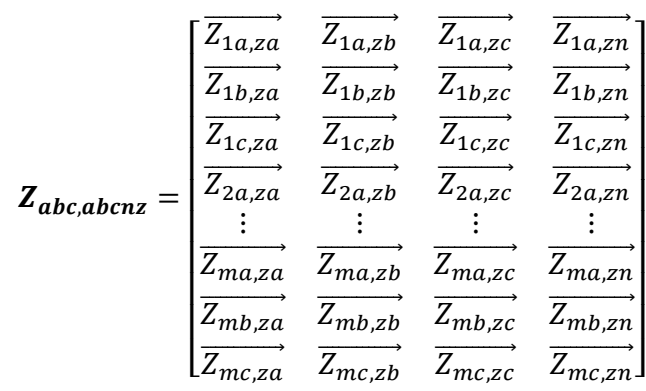

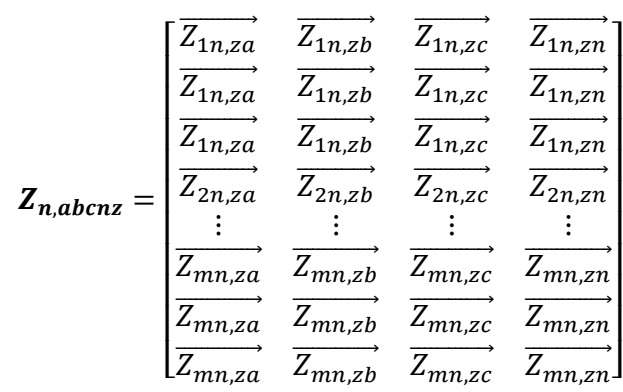

Constraint 6: + di $i_{0}$ action for $I_{z, \text { imag }}^{0}$ of $D G z$

$$
\begin{gathered}
\max \left(\boldsymbol{V}_{p h-n}+\left(\boldsymbol{Z}_{\boldsymbol{a b c \boldsymbol { a b } c n z}}-\boldsymbol{Z}_{n, \boldsymbol{a b c n z}}\right) \cdot\left[\begin{array}{c}
1 \\
1 \\
1 \\
-3
\end{array}\right] \cdot j \cdot d i_{0}\right)-\min \left(\boldsymbol{V}_{p h-n}+\right. \\
\left.\left(\boldsymbol{Z}_{\boldsymbol{a b c , a b c n z}}-\boldsymbol{Z}_{n, \boldsymbol{a b c n z}}\right) \cdot\left[\begin{array}{c}
1 \\
1 \\
1 \\
-3
\end{array}\right] \cdot j \cdot d i_{0}\right)<V_{\text {limit }}^{\text {upper }}-V_{\text {limit }}^{\text {lower }}
\end{gathered}
$$

Constraint 7: -dp action for $P_{z}^{+}$of $D G z$

Constraint 8: $-d q$ action for $Q_{z}^{+}$of $D G z$

Constraint 9: -di_action for $I_{z, \text { real }}^{-}$of $D G z$

Constraint 10: - di_ action for $I_{z, i m a g}^{-}$of $D G z$

Constraint 11: $-d i_{0}$ action for $I_{z, \text { real }}^{0}$ of $D G z$

Constraint 12: $-d i_{0}$ action for $I_{z, \text { imag }}^{0}$ of $D G z$

Constraints 7, 8, 9, 10, 11 and 12 are obtained in a similar sense as the constraints $1,2,3,4,5$ and 6 , respectively.

\section{D) Steps of the Proposed Approach}

The proposed algorithm is executed in the following steps: Step 1: Define the step values of control variables $\left(d p, d q, d i_{-}\right.$ ,$\left.d i_{0}\right)$. Small values of $d p, d q, d i_{-}, d i_{0}$ lead to increased accuracy of the final solution but the convergence speed is sacrificed. Moreover, the negative- and zero-sequence currents of all DGs are initially set to zero. The positive- sequence powers are arbitrarily set with initial values so that the power limits of all DGs (and the slack bus) are satisfied. Step 2: The power flow is executed.

Step 3: Based on the power flow results, we update the Sens matrix of (10). Subsequently, we construct the $\boldsymbol{S e n s}_{\text {mod }}$ matrix of (11)

Step 4: The proposed SBCM method is executed. More specifically, based on the power flow result of step 2, we search for the best action ( \pm rand $d p, \pm$ rand $d q$, \pm rand $\cdot d i_{-}$, \pm rand $\cdot d i_{0}$ ) between the DGs so that the cost function of (12) is minimized and the constraints of Section III-C are satisfied. The term 'rand' above is a uniformly distributed random number between $[0,1]$ and it is multiplied with the step values of control variables in order to prevent the SBCM algorithm from sticking to contrasting actions from the same DG e.g $+\mathrm{dp} \rightarrow-\mathrm{dp} \rightarrow+\mathrm{dp}$.

Step 5: Update the power or current of one DG based on the optimal result of the previous step.

Step 6: Update the slack voltage by $d V_{\text {slack }}$, as follows:

$$
d V_{\text {slack }}=V_{\text {nom }}-\frac{\max \left(V_{p h-n}\right)+\min \left(V_{p h-n}\right)}{2}
$$

where $V_{\text {nom }}, \max \left(\boldsymbol{V}_{p h-n}\right), \min \left(\boldsymbol{V}_{p h-n}\right)$ is the nominal, maximum and minimum phase-to-neutral voltage of the network, respectively. In this way, the phase-to-neutral voltages of all buses are always inside their limits provided that the constraints (35), (40)-(43), (46) are fulfilled.

Step 7: Go to Step 2 until the magnitudes of the three-phase and neutral current of the switched line are not further reduced. Step 8: Output the $P_{z}^{+}, Q_{z}^{+}, I_{z, \text { real }}^{-}, I_{z, \text { imag }}^{-}, I_{z, \text { real }}^{0}, I_{z, \text { imag }}^{0}$ for each DG $z$ (including the slack). Calculate the positivesequence droop curves for each DG $z$ (including the slack), from $P_{z}^{+}, Q_{z}^{+}$, as explained in section IV.A.

Step 9: Apply the output values to the control scheme of each DG $z$, as explained in section IV.B.

\section{PRACTICAL IMPLEMENTATION OF THE PROPOSED METHOD}

In this section the practical implementation of the proposed SBCM approach is explained in an inverter-based DG (IBDG). Firstly, we explain how the parameters of the droop equations are obtained, based on the output commands of the SBCM method. Then, we provide the schematic diagram of a droop-controlled IBDG, in order to clarify how are the output commands of the proposed method applied in the control system of IBDGs.

\section{A) Calculation of droop equations of IBDGs during the switching action.}

In unbalanced islanded MGs, droop equations are applied on the positive-sequence power and voltage of IBDGs. The droop equations of IBDG $z$ are given by equations (48)-(49) [15] [16]:

$$
\begin{aligned}
\left|\overrightarrow{V_{z}^{+}}\right| & =\left|\overrightarrow{V_{z, r e f}^{+}}\right|-K_{q z} \cdot Q_{z}^{+} \\
f & =f_{\text {ref }}-K_{p z} \cdot P_{z}^{+}
\end{aligned}
$$


where $\overrightarrow{V_{z}^{+}}, \overrightarrow{V_{z}^{+}, r e f}, K_{q z}, K_{p z}, Q_{z}^{+}, P_{z}^{+}, f_{\text {ref }}, f$ is the positivesequence voltage, reference voltage, reactive and active power droop gains, positive-sequence reactive and active power, reference frequency and frequency of DG $z$, respectively.

For DG $z, Q_{z}^{+}$and $\overrightarrow{V_{z}^{+}}$have been calculated from the SBCM and power flow, respectively. In order to form the droop equation (48) for DG $z$, at the time instant of switching action, $K_{q z}$ and $\left|\overrightarrow{V_{z, r e f}^{+}}\right|$are arbitrarily selected such that (48) is satisfied, for the calculated $Q_{z}^{+}$and $\overrightarrow{V_{z}^{+}}$. This process is followed for all DGs, including the one assumed as slack bus. In this way, the droop operation of MGs is retained during the switching action, for all DGs, including the slack one.

A similar approach is followed to form (49) for each DG $z$. For example, assuming that there exist 3 DGs $i, j, z$ (including the slack DG), $P_{i}^{+}, P_{j}^{+}, P_{z}^{+}$have been calculated from the SBCM method. Since the frequency $f$ is the same for all DGs, $f_{r e f}, K_{p i}, K_{p j}, K_{p z}$ are arbitrarily selected such that equation system $f_{r e f}-K_{p i} \cdot P_{i}^{+}=f_{r e f}-K_{p j} \cdot P_{j}^{+}=f_{r e f}-K_{p z} \cdot P_{z}^{+}$ is satisfied.

\section{B) Implementation of the proposed SBCM method on the control system of IBDGs}

Currently, IBDGs are usually controlled in the rotating dq0 reference frame. The three sequence components are separately treated in three different dq frames, rotating with different rotational speeds [19]-[22].

The proposed SBCM algorithm outputs the droop curves of DGs in the positive-sequence component (see section IV.A) as well as the negative- and zero sequence currents. These outputs can be easily incorporated into the existing DG control schemes. The schematic diagram of the implementation of the proposed approach in a practical IBDG control scheme is shown in Fig. 3 (at the end of the paper). The positivesequence power is measured and accordingly, based on the droop equations, form the positive-sequence voltage of IBDG in the positive-sequence dq reference frame. The real and imaginary components of negative-sequence currents correspond to $I_{d, n}^{*}, I_{q, n}^{*}$, in Fig. 3, respectively. Finally, the real and imaginary components of zero-sequence currents correspond to $I_{d, h}^{*}, I_{q, h}^{*}$, in Fig. 3, respectively.

\section{Simulation Results}

The performance of the proposed SBCM approach is investigated using a balanced 33-bus as well as the unbalanced IEEE 8500-node network.

\section{A) 33-bus Balanced Network}

Firstly, a comparison of the proposed SBCM approach against the mixed-integer nonlinear programming (MINP) approach of [11] is attempted in this sub-section using the 33bus network shown in Fig. 11 of [11]. Eight switching actions are executed in a sequence, as follows: 1) closing line 35,2 ) opening line 10,3) closing line 34,4) opening line 12,5) closing line 36,6 ) opening line 30,7 ) closing line 37,8 ) opening line 22. Details about the network are provided in
[11]. The DG limits are shown in Table I. The voltage limits were considered $\pm 5 \%$ of the nominal voltage.

Fig. 4 depicts the per unit value of the switched power of the eight switching actions using the proposed SBCM method, the MINP approach of [11] and the case where no minimization of the switched power is realized. In the last case, the DGs operate in droop control achieving a proportional load sharing. It is clarified that the switching actions are executed in a row, as shown in Fig. 4, and therefore, the structure of the network is successively changed after each switching action. As shown the results of the proposed SBCM method are almost identical with the MINP method of [11]. Both methods achieve a significant power reduction in all switching actions compared to the case "without minimization". The slack voltage and power of DG 1 , the power of the switched line, and the powers of DG 2 and 3 are depicted in Table II. In Table II, the powers of DGs that have reached their limits are depicted with bold.

TABLE I

POWER LIMITS OF DGS FOR THE 33-BUS NETWORK (IN PU)

\begin{tabular}{|c|c|c|c|c|c|}
\hline & Min P & Max P & Min Q & Max Q & Max S \\
\hline DG 1, Bus 1 & 0 & 1.92 & 0 & 1.44 & 2.4 \\
\hline DG 2, Bus 18 & 0 & 1.6 & 0 & 1.2 & 2 \\
\hline DG 3, Bus 24 & 0 & 1.92 & 0 & 1.44 & 2.4 \\
\hline
\end{tabular}

TABLE II

Voltage of Slack Bus, Power of Switched Line, Active and Reactive POWER OF DGs FOR All SWITCHING ACTIONS USING THE PROPOSED METHOD.

\begin{tabular}{|c|c|c|c|c|c|c|}
\hline \begin{tabular}{|l|} 
Switching \\
action's \\
number
\end{tabular} & $\begin{array}{c}\text { Switching } \\
\text { action }\end{array}$ & $\begin{array}{l}\text { Slack } \\
\text { Voltage } \\
\text { (DG1) }\end{array}$ & $\begin{array}{c}\text { Switched } \\
\text { Power }\end{array}$ & $\begin{array}{c}\text { Slack Power } \\
\text { (DG 1) } \\
(\mathbf{p}, \mathbf{q})\end{array}$ & $\begin{array}{l}\text { Power } \\
\text { DG } 2 \\
(p, q)\end{array}$ & $\begin{array}{c}\text { Power } \\
\text { DG } 3 \\
(p, q)\end{array}$ \\
\hline 1 & $\begin{array}{l}\text { Closing } \\
\text { line } 35\end{array}$ & 1.0061 & $1.8 \cdot 10^{-5}$ & $\begin{array}{l}(1.4079 \\
0.8313)\end{array}$ & $\begin{array}{l}(1.1333, \\
0.7357)\end{array}$ & $\begin{array}{l}(1.2733 \text {, } \\
0.8032)\end{array}$ \\
\hline 2 & $\begin{array}{c}\text { Opening } \\
\text { line } 10\end{array}$ & 1.0246 & 0.1109 & $\begin{array}{l}(1.8400, \\
0.9348)\end{array}$ & $\begin{array}{c}(0.0659, \\
0)\end{array}$ & $\begin{array}{l}(1.9193, \\
1.4400)\end{array}$ \\
\hline 3 & $\begin{array}{l}\text { Closing } \\
\text { line } 34\end{array}$ & 1.0262 & 0.0648 & $\begin{array}{c}\mathbf{( 1 , 9 1 9 5}, \\
0,9294)\end{array}$ & $\begin{array}{c}(0.1437, \\
0)\end{array}$ & $\begin{array}{l}(1.7569, \\
\mathbf{1 . 4 3 9 9})\end{array}$ \\
\hline 4 & $\begin{array}{c}\text { Opening } \\
\text { line } 12\end{array}$ & 1.0223 & $9.7 \cdot 10^{-6}$ & $\begin{array}{l}(1.6882, \\
0.9611)\end{array}$ & $\begin{array}{l}(0.8213, \\
0.5998)\end{array}$ & $\begin{array}{l}(1.2855, \\
0.7918)\end{array}$ \\
\hline 5 & $\begin{array}{l}\text { Closing } \\
\text { line } 36\end{array}$ & 1.0236 & 0.1958 & $\begin{array}{l}(1.6112, \\
0.9293)\end{array}$ & $\begin{array}{c}(0.2911, \\
0)\end{array}$ & $\begin{array}{c}(1.9197, \\
1.44)\end{array}$ \\
\hline 6 & $\begin{array}{l}\text { Opening } \\
\text { line } 30\end{array}$ & 1.0171 & $7.7 \cdot 10^{-6}$ & $\begin{array}{c}(1.0866, \\
1.2259)\end{array}$ & $\begin{array}{l}(0.7795, \\
0.1299)\end{array}$ & $\begin{array}{l}(\mathbf{1 . 9 1 9 7}, \\
0.9824)\end{array}$ \\
\hline 7 & $\begin{array}{l}\text { Closing } \\
\text { line } 37\end{array}$ & 0.9864 & 0.2160 & $\begin{array}{c}\mathbf{( 1 . 9 1 9 5}, \\
1.1642)\end{array}$ & $\begin{array}{c}(1.5999 \\
1.2000\end{array}$ & $\begin{array}{c}(0.2809, \\
0)\end{array}$ \\
\hline 8 & $\begin{array}{c}\text { Opening } \\
\text { line } 22\end{array}$ & 0.9986 & $7.2 \cdot 10^{-5}$ & $\begin{array}{l}(1.3675, \\
0.5595)\end{array}$ & $\begin{array}{l}(1.0762, \\
0.8278)\end{array}$ & $\begin{array}{l}(1.3049, \\
0.9320)\end{array}$ \\
\hline
\end{tabular}

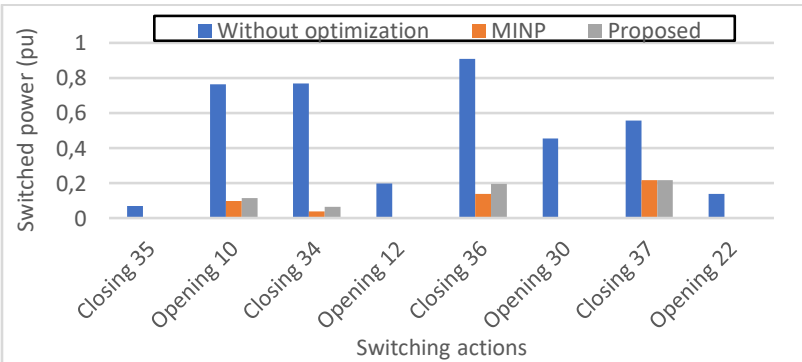

Fig. 4. Power of the switched line for the 8 examined switching actions of the 33-bus network, using the proposed SBCM approach, the MINP of [11] and the case where no minimization of the switched power is realized.

\section{B) IEEE 8500-Node Network}

The topology of the unbalanced IEEE 8500 node network is shown in Fig. 5. It is a 4-wire multi-grounded MV network with a phase-to-neutral nominal voltage $7.2 \mathrm{kV}$ and total loading 10.7 MW and 2.7 MVar [18]. Data about the loads and 
lines are provided in [18]. The neutral is assumed to be grounded every 100 buses with a grounding resistance 10 Ohm. The network is considered islanded, supplied by 5 DGs, as shown in Fig. 5. Data about the DGs are given in Table III.

The network is assumed reconfigurable with 8 switching actions, as referred in Table IV. The efficiency of the proposed SBCM approach in the aforementioned switching actions is compared against the case, where no minimization of the line current is performed. In the last case DGs operate in droop control with a proportional power sharing. The magnitudes of the phase currents of the switched line for both cases are depicted in Table IV. As shown, the proposed SBCM approach achieves a significant current reduction in all phases, thus preventing the negative impacts of the line switching.

The magnitudes of the three phase and neutral currents throughout the iterative execution of the proposed SBCM method are illustrated in Fig. 6 for the switching action "Opening isolation switch 3" of Table IV. The horizontal axis depicts the number of SBCM executions. The current is almost nullified after around 70 SBCM executions. As explained in section III, each SBCM corresponds to one power flow execution, namely to around 10 power flow iterations. Therefore, the proposed approach requires in total around 700 power flow iterations $(70 \cdot 10)$.

The optimal positive-sequence active and reactive power of DGs in order to smooth the line-switching action "Opening isolation switch 3" are shown in Figs. 7 and 8, respectively. As shown, the power limits of Table III are always satisfied throughout the execution of the proposed SBCM approach

The magnitudes of the optimal negative- and zero-sequence currents of DGs are depicted in Figs. 9 and 10, respectively. As shown, DGs inject a significant amount of negative- and zero-sequence current to compensate the unbalance loading of the network. In contrast to MINP method of [11], in which the authors assumed a balanced network optimizing only the positive-sequence components of DGs, the proposed SBCM approach is able to optimize also the negative and zerosequence components.

The phase-to-neutral voltage of DG 1 (it is considered the slack bus in the power flow and has always balanced phaseto-neutral voltage) along with the maximum and minimum phase-to-neutral voltage of the network are depicted in Fig. 11. As shown, the deviation between maximum and minimum voltage remains always below $1440 \mathrm{~V}( \pm 10 \%$ of $7200 \mathrm{~V})$, which is the bandwidth imposed by the voltage constraints (35), (40)-(43), (46). The slack voltage is updated after each power flow execution according to (47), to maintain all phaseto-neutral voltages of the network between $0.9 \cdot 7200 \mathrm{~V}$ and $1.1 \cdot 7200 \mathrm{~V}$.

Finally, Table V depicts a comparison between the proposed SBCM method (optimizing the positive sequence powers as well as the negative- and zero-sequence currents), against the simplistic case, where only the positive-sequence powers are optimized. The last case represents the approach of [11], where the authors consider a balanced network, assuming that the negative- and zero-sequence currents of DGs are zero, and therefore, the optimization is performed only on the positive-sequence powers. In the table, only the three switching actions with the highest currents are depicted (switching action's number 2, 6, 8 of Table IV). As shown, in the two out of three cases, the single-phase approach of [11] does not effectively minimize the line currents, in contrast to the proposed SBCM method, which achieves a large current reduction in all cases.

TABLE III

POWER LIMITS OF DGS FOR THE 8500-NODE NETWORK

\begin{tabular}{|c|c|c|c|c|c|}
\hline & Min P & Max P & Min Q & Max Q & Max S \\
\hline DG 1 (SLACK) & $0 \mathrm{MW}$ & $3 \mathrm{MW}$ & $-1 \mathrm{MVAR}$ & $1 \mathrm{MVAR}$ & $3.16 \mathrm{MVA}$ \\
\hline DG 2 & $0 \mathrm{MW}$ & $3 \mathrm{MW}$ & $-1 \mathrm{MVAR}$ & $1 \mathrm{MVAR}$ & $3.16 \mathrm{MVA}$ \\
\hline DG 3 & $0 \mathrm{MW}$ & $3 \mathrm{MW}$ & $-1 \mathrm{MVAR}$ & $1 \mathrm{MVAR}$ & $3.16 \mathrm{MVA}$ \\
\hline DG 4 & $0 \mathrm{MW}$ & $3 \mathrm{MW}$ & $-1 \mathrm{MVAR}$ & $1 \mathrm{MVAR}$ & $3.16 \mathrm{MVA}$ \\
\hline DG 5 & $0 \mathrm{MW}$ & $3 \mathrm{MW}$ & $-1 \mathrm{MVAR}$ & $1 \mathrm{MVAR}$ & $3.16 \mathrm{MVA}$ \\
\hline
\end{tabular}

TABLE IV

PhaSE CURRENTS THRough THE SWITCHED LINES USING THE PROPOSED SBCM AND THE CASE "WITHOUT MINIMIZATION"

\begin{tabular}{|c|c|c|c|}
\hline $\begin{array}{l}\text { Switching } \\
\text { action's } \\
\text { number }\end{array}$ & Switching action & $\begin{array}{c}\text { Current } \\
\text { without optimization }\end{array}$ & $\begin{array}{l}\text { Current using the } \\
\text { proposed SBCM } \\
\text { approach }\end{array}$ \\
\hline 1 & \begin{tabular}{|c|} 
Connecting \\
connection points \\
$1-2$
\end{tabular} & $\begin{array}{l}I_{a}=19.3661 \mathrm{~A} \\
I_{b}=11.3035 \mathrm{~A} \\
I_{c}=13.1123 \mathrm{~A}\end{array}$ & $\begin{aligned} I_{a} & =0.6225 \mathrm{~A} \\
I_{b} & =0.9737 \mathrm{~A} \\
I_{c} & =3.5056 \mathrm{~A}\end{aligned}$ \\
\hline 2 & $\begin{array}{c}\text { Opening isolation } \\
\text { switch } 1\end{array}$ & $\begin{aligned} I_{a} & =88.5474 \mathrm{~A} \\
I_{b} & =93.4523 \mathrm{~A} \\
I_{c} & =20.8382 \mathrm{~A}\end{aligned}$ & $\begin{array}{l}I_{a}=0.5143 \mathrm{~A} \\
I_{b}=2.6917 \mathrm{~A} \\
I_{c}=0.7417 \mathrm{~A}\end{array}$ \\
\hline 3 & \begin{tabular}{|c|} 
Connecting \\
connection points \\
$2-3$
\end{tabular} & $\begin{array}{c}I_{a}=15.7981 \mathrm{~A} \\
I_{b}=32.0682 \mathrm{~A} \\
I_{c}=3.3856 \mathrm{~A}\end{array}$ & $\begin{array}{l}I_{a}=1.2503 \mathrm{~A} \\
I_{b}=6.4557 \mathrm{~A} \\
I_{c}=0.8714 \mathrm{~A}\end{array}$ \\
\hline 4 & $\begin{array}{c}\text { Opening isolation } \\
\text { switch } 2\end{array}$ & $\begin{array}{c}I_{a}=25.3095 \mathrm{~A} \\
I_{b}=7.2565 \mathrm{~A} \\
I_{c}=24.5085 \mathrm{~A}\end{array}$ & $\begin{aligned} I_{a} & =0.6923 \mathrm{~A} \\
I_{b} & =4.2211 \mathrm{~A} \\
I_{c} & =1.6958 \mathrm{~A}\end{aligned}$ \\
\hline 5 & \begin{tabular}{|c|} 
Connecting \\
connection points \\
$3-4$
\end{tabular} & $\begin{array}{c}I_{a}=8.4482 \mathrm{~A} \\
I_{b}=18.8017 \mathrm{~A} \\
I_{c}=20.3190 \mathrm{~A}\end{array}$ & $\begin{aligned} I_{a} & =2.2938 \mathrm{~A} \\
I_{b} & =0.1831 \mathrm{~A} \\
I_{c} & =2.0323 \mathrm{~A}\end{aligned}$ \\
\hline 6 & $\begin{array}{c}\text { Opening isolation } \\
\text { switch } 4\end{array}$ & $\begin{array}{l}I_{a}=106.2018 \mathrm{~A} \\
I_{b}=101.2145 \mathrm{~A} \\
I_{c}=106.2007 \mathrm{~A}\end{array}$ & $\begin{array}{c}I_{a}=8.6389 \mathrm{~A} \\
I_{b}=16.7107 \mathrm{~A} \\
I_{c}=11.8661 \mathrm{~A}\end{array}$ \\
\hline 7 & \begin{tabular}{|c|} 
Connecting \\
connection points \\
$4-5$
\end{tabular} & $\begin{aligned} I_{a} & =11.0360 \mathrm{~A} \\
I_{b} & =15.6320 \mathrm{~A} \\
I_{c} & =42.4365 \mathrm{~A}\end{aligned}$ & $\begin{aligned} I_{a} & =0.2644 \mathrm{~A} \\
I_{b} & =0.7860 \mathrm{~A} \\
I_{c} & =0.2184 \mathrm{~A}\end{aligned}$ \\
\hline 8 & $\begin{array}{c}\text { Opening isolation } \\
\text { switch } 3\end{array}$ & $\begin{array}{c}I_{a}=107.2988 \mathrm{~A} \\
I_{b}=107.8948 \mathrm{~A} \\
I_{c}=12.4962 \mathrm{~A}\end{array}$ & $\begin{aligned} I_{a} & =3.8054 \mathrm{~A} \\
I_{b} & =1.1401 \mathrm{~A} \\
I_{c} & =0.9511 \mathrm{~A}\end{aligned}$ \\
\hline
\end{tabular}

TABLE $\mathrm{V}$

PHASE CURRENTS THROUGH THE SWITCHED LINES USING THE PROPOSED SBCM AND THE CASE WHERE ONLY THE POSITIVE SEQUENCE ACTIVE AND REACTIVE POWERS ARE MINIMIZED [11]

\begin{tabular}{|c|c|c|c|}
\hline $\begin{array}{c}\text { Switching } \\
\text { action }\end{array}$ & $\begin{array}{c}\text { Current } \\
\text { without optimization }\end{array}$ & $\begin{array}{c}\text { Current using the } \\
\text { proposed SBCM } \\
\text { approach }\end{array}$ & $\begin{array}{c}\text { Currents optimizing } \\
\text { only the positive } \\
\text { sequence powers } \\
\text { [11] }\end{array}$ \\
\hline $\begin{array}{c}\text { Opening } \\
\text { isolation switch }\end{array}$ & $I_{a}=88.5474 \mathrm{~A}$ & $I_{a}=0.5143 \mathrm{~A}$ & $I_{a}=29.36 \mathrm{~A}$ \\
1 & $I_{b}=93.4523 \mathrm{~A}$ & $I_{b}=2.6917 \mathrm{~A}$ & $I_{b}=38.72 \mathrm{~A}$ \\
\hline Opening & $I_{a}=106.2018 \mathrm{~A}$ & $I_{c}=0.7417 \mathrm{~A}$ & $I_{c}=93.73 \mathrm{~A}$ \\
\hline isolation switch & $I_{b}=101.2145 \mathrm{~A}$ & $I_{b}=12.7107 \mathrm{~A}$ & $I_{a}=5.24 \mathrm{~A}$ \\
4 & $I_{c}=106.2007 \mathrm{~A}$ & $I_{c}=11.8661 \mathrm{~A}$ & $I_{b}=15.00 \mathrm{~A}$ \\
\hline Opening & $I_{a}=107.2988 \mathrm{~A}$ & $I_{a}=3.8054 \mathrm{~A}$ & $I_{a}=13.18 \mathrm{~A}$ \\
isolation switch & $I_{b}=107.8948 \mathrm{~A}$ & $I_{b}=1.1401 \mathrm{~A}$ & $I_{b}=11.59 \mathrm{~A}$ \\
3 & $I_{c}=12.4962 \mathrm{~A}$ & $I_{c}=0.9511 \mathrm{~A}$ & $I_{c}=83.50 \mathrm{~A}$ \\
\hline
\end{tabular}




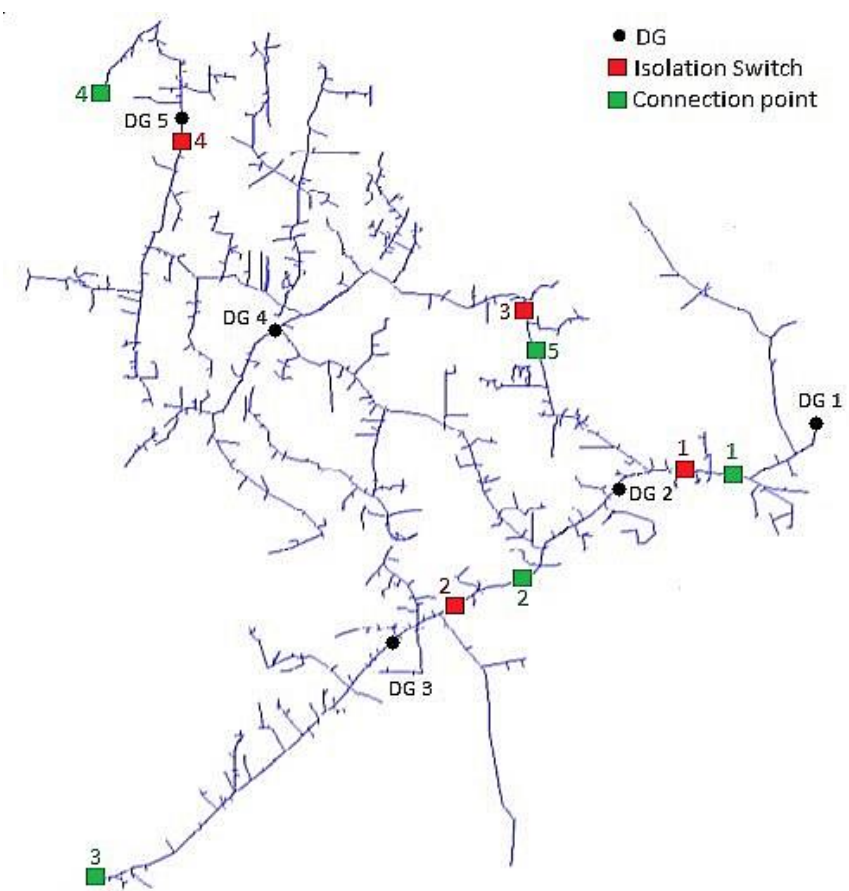

Fig. 5 Modified IEEE 8500-Node network operating in islanded mode.

\section{C) Computation time of the proposed SBCM approach}

The computation time of the proposed SBCM approach is compared in Fig. 12 against the MINP method of [11], for the 8 switching actions (see Table II) of the balanced 33-Bus network. All simulations were executed in a PC with a 64-bit Intel Core i7, 3.4GHz CPU and 16GB RAM. As shown, the proposed SBCM approach is faster in all examined switching actions.

When applied in the unbalanced 8500-node network, the proposed SBCM approach needs 300 to 700 power flow iterations to converge, depending on the switching action. Since the execution time of each iteration is around 0.2 seconds, the total execution time of the proposed approach ranges from 60 to 140 seconds. Due to the large size of the network and the precise three-phase (5-wire a-b-c-n-g) representation of the proposed SBCM approach, we believe that this time is reasonably low, making the proposed approach an important tool for smoothing the switching actions of unbalanced islanded MGs.

Finally, it is clarified that the MINP method of [11] is not applicable in unbalanced MGs, and thus, a comparison against the proposed method is not possible in the unbalanced 8500node MG.

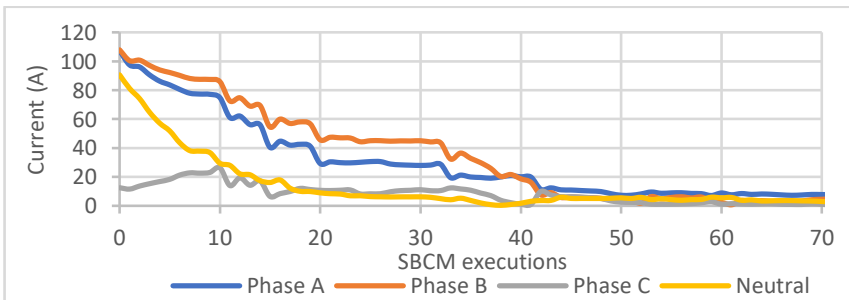

Fig. 6. Magnitude of the three-phase and neutral currents of isolation switch 3 throughout the execution of the proposed SBCM approach.

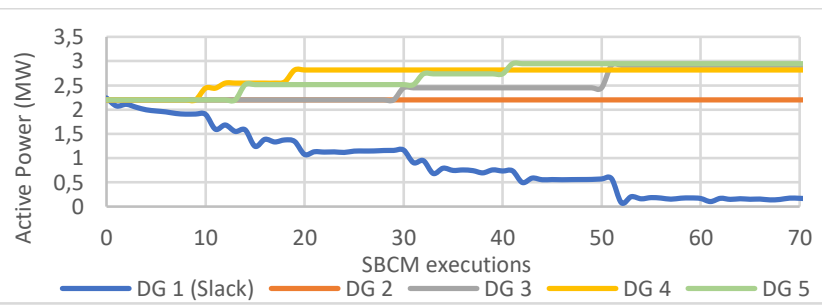

Fig. 7. Positive-sequence active power of DGs throughout the execution of the proposed SBCM approach.

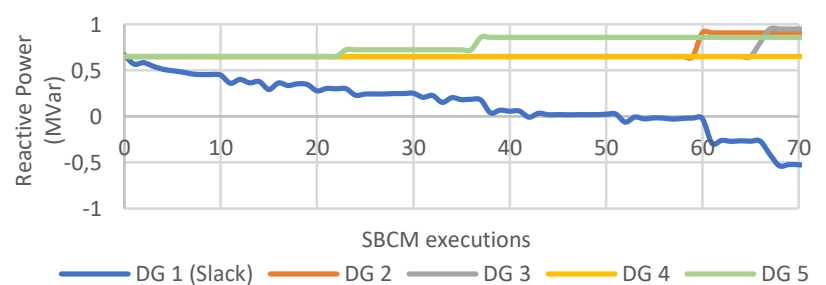

Fig. 8. Positive-sequence reactive power of DGs throughout the execution of the proposed SBCM approach.

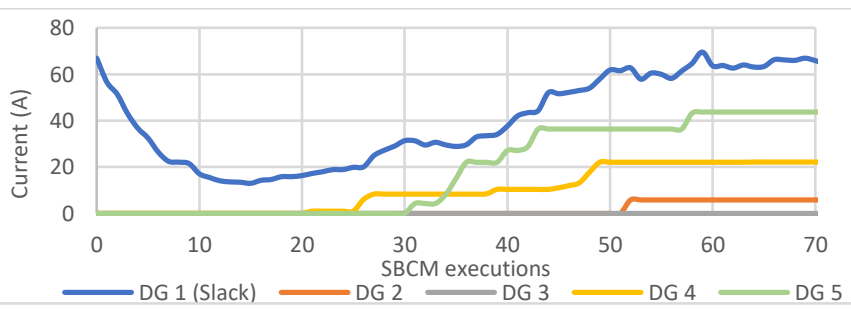

Fig. 9. Magnitude of the negative-sequence current of DGs throughout the execution of the proposed SBCM approach.

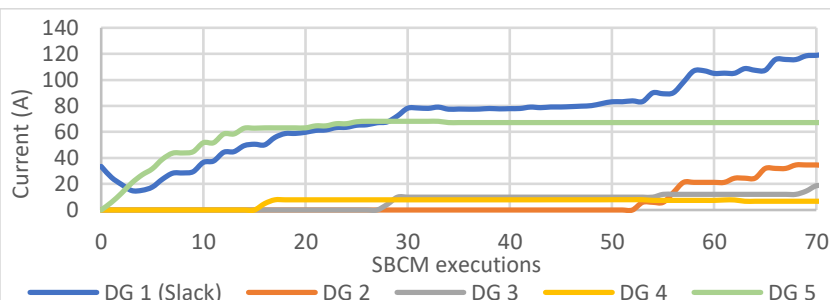

Fig. 10. Magnitude of the zero-sequence current of DGs throughout the execution of the proposed SBCM approach.

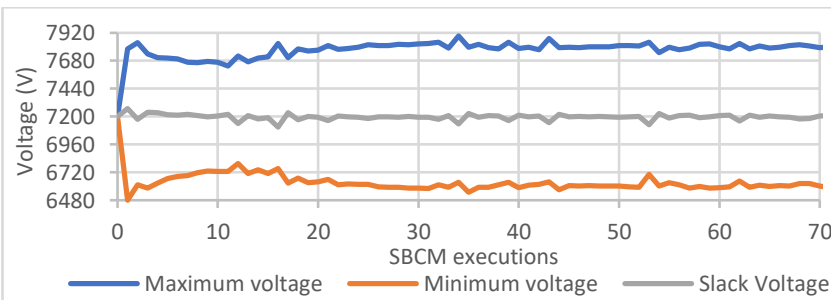

Fig. 11. Maximum and minimum phase-to-neutral voltage of the network as well as phase-to-neutral voltage of slack bus, throughout the execution of the proposed SBCM approach.

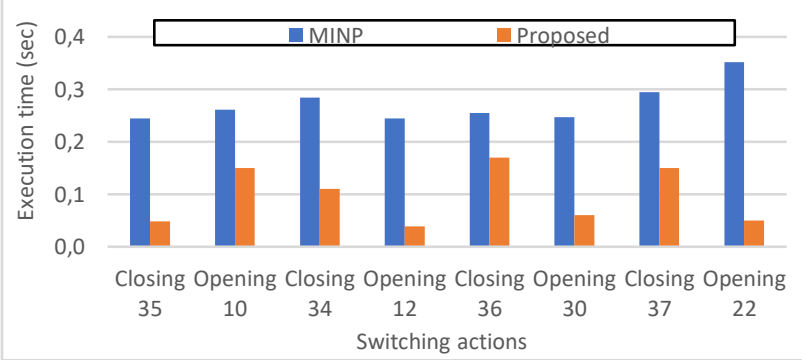

Fig. 12 Computation time of the 8 examined switching actions of the 33-bus network, using the proposed SBCM approach and the MINP of [11]. 


\section{CONCLUSION}

Network reconfiguration is an effective strategy applied in islanded MGs to optimize their performance. This paper proposes a three-phase sensitivity-based method to mitigate the negative impacts of the line-switchings in unbalanced low voltage and medium voltage islanded networks. The proposed sensitivity-based method outputs the optimal positivesequence powers as well as the negative- and zero-sequence currents of all DGs so that the current flowing through the three-phases and neutral of the switched line is minimum. Simulations were executed in a balanced islanded 33-bus as well as in the unbalanced IEEE 8500-node networks to validate the performance of the method.

\section{REFERENCES}

[1] R. S. Rao, K. Ravindra, K. Satish, and S. V. L. Narasimham, "Power loss minimization in distribution system using network reconfiguration in the presence of distributed generation," IEEE Trans. Power Syst., vol. 28, no. 1, pp. 317-325, 2013.

[2] E. Dall'Anese and G. B. Giannakis, "Risk-constrained microgrid reconfiguration using group sparsity," IEEE Trans. Sustain. Energy, vol. 5, no. 4, pp. 1415-1425, 2014.

[3] S. Thakar, A. S. Vijay, and S. Doolla, "Effect of P-Q Limits on microgrid reconfiguration: A capability curve perspective," IEEE Trans. Sustain. Energy, vol. 11, no. 3, pp. 2040-2048, 2020.

[4] M. M. A. Abdelaziz, H. E. Farag, and E. F. El-Saadany, "Optimum reconfiguration of droop-controlled islanded microgrids," IEEE Trans. Power Syst., vol. 31, no. 3, pp. 2144-2153, 2016.

[5] M. H. Amirioun, F. Aminifar, and H. Lesani, "Resilience-oriented proactive management of microgrids against windstorms," IEEE Trans. Power Syst., vol. 33, no. 4, pp. 4275-4284, 2018.

[6] A.Kavousi-Fard, A.Khodaei, "Efficient integration of plug-in electric vehicles via reconfigurable microgrids", Energy, vol. 111, 653-663, 2016.

[7] S. Gazijahani, J. Salehi, "Integrated DR and reconfiguration scheduling for optimal operation of microgrids using Hongs point estimate method," Int. J. Electr. Power Energy Syst., vol. 99, 481-492, 2018.

[8] M. Khederzadeh and A. Beiranvand, "Identification and prevention of cascading failures in autonomous microgrid," IEEE Syst. J., vol. 12, no. 1, pp. 308-315, Mar. 2018.
[9] Sushrut Thakar, Vijay A.S, Suryanarayana Doolla, "System reconfiguration in microgrids," Sustain. Energy, Grids Netw., 100191, vol. $17,2019$.

[10] Z. Li, S. Jazebi, and F. de León, "Determination of the optimal switching frequency for distribution system reconfiguration," IEEE Trans. Power Del., vol. 32, no. 4, pp. 2060-2069, 2017.

[11] W. T. El-Sayed; H. Farag, H. H. Zeineldin, E. F. El-Saadany, "Dynamic transitional droops for seamless line-switching in islanded microgrids", IEEE Trans. Power Syst., (Early Access), doi: 10.1109/TPWRS.2021.3069852.

[12] "IEEE Guide for conducting distribution impact studies for distributed resource interconnection," IEEE Std 1547.7-2013. pp. 1-137, 2014.

[13] "IEEE Guide for Service to Equipment Sensitive to Momentary Voltage Disturbances," IEEE Std 1250-1995. p. 0 1, 1995

[14] N. Soni, S. Doolla, and M. C. Chandorkar, "Improvement of transient response in microgrids using virtual inertia," IEEE Trans. Power Del. vol. 28 , no. 3, pp. 1830-1838, 2013.

[15] E. E. Pompodakis, G. C. Kryonidis, C. S. Demoulias, M. C. Alexiadis, "A generic power flow algorithm for unbalanced islanded hybrid AC/DC microgrids," IEEE Trans. Power Syst. (Early Access), doi: 10.1109/TPWRS.2020.3012815, July 2020.

[16] E. E. Pompodakis, G. C. Kryonidis, M. C. Alexiadis, "A comprehensive load flow approach for grid-connected and islanded AC microgrids", IEEE Trans. Power Syst., vol. 35, no. 2, pp. 1143-1155, 2020.

[17] E. E. Pompodakis et al., "Photovoltaic systems in low-voltage networks and overvoltage correction with reactive power control," IET Renew. Power Gener., vol. 10, no. 3, pp. 410-417, 2016.

[18] IEEE PES. (2021, May.) Distribution test feeders, "8500-Node Test Feeder". [Online]. Available: http://www.ewh.ieee.org/soc/pes/dsacom/testfeeders/index.html

[19] Vechiu et al., "Control of four leg inverter for hybrid power system applications with unbalanced load", Energy Conversion and Management, 2119-2128, 2007.

[20] H. Karimi et. al., "Negative-Sequence Current Injection for Fast Islanding Detection of a Distributed Resource Unit", IEEE Transactions on Power electronics, vol. 23, No 1, 2008

[21] H. Shi et. al., "Control strategy for microgrid under three-phase unbalance condition", J. Mod. Power Syst. Clean Energy, 4(1):94-102, 2016.

[22] X.Zhou et. al. "Four-Leg Converters With Improved Common Current Sharing and Selective Voltage-Quality Enhancement for Islanded Microgrids", Transactions on Power Delivey, vol. 31, No. 2, 2016

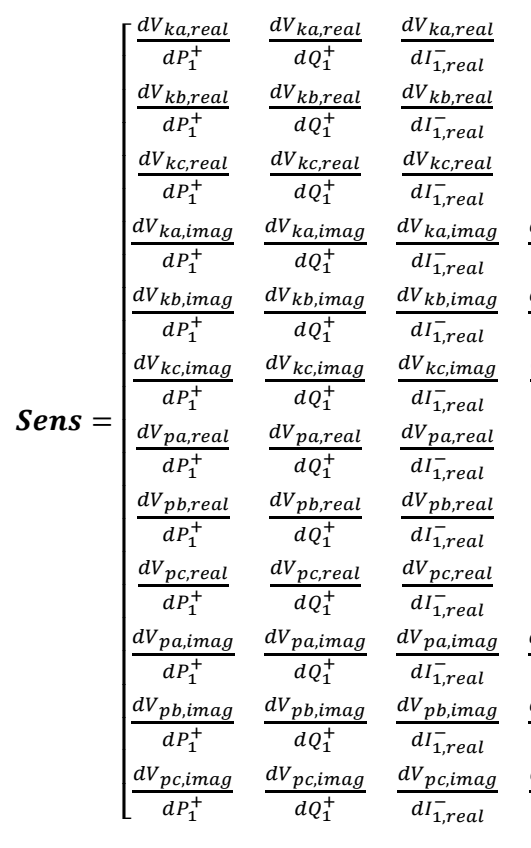

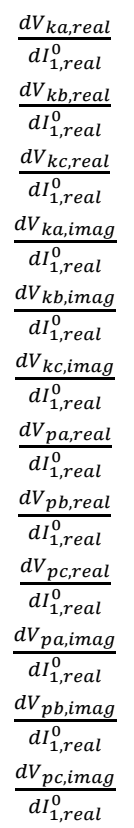

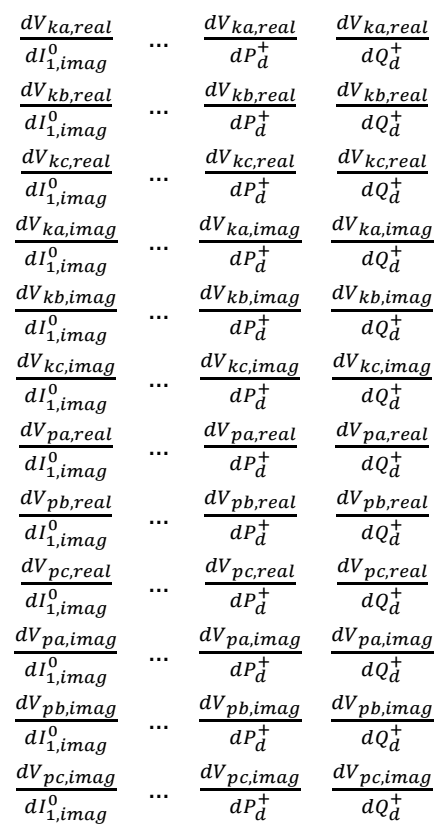

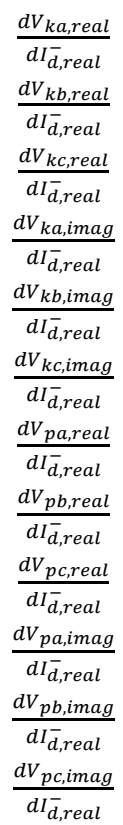

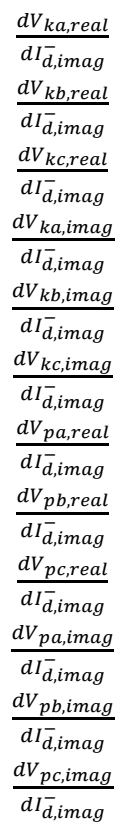

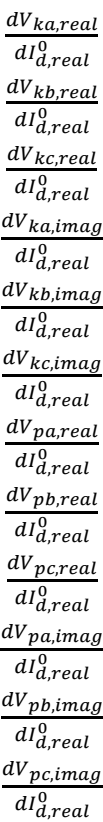

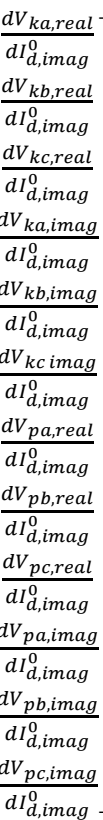

(10) 


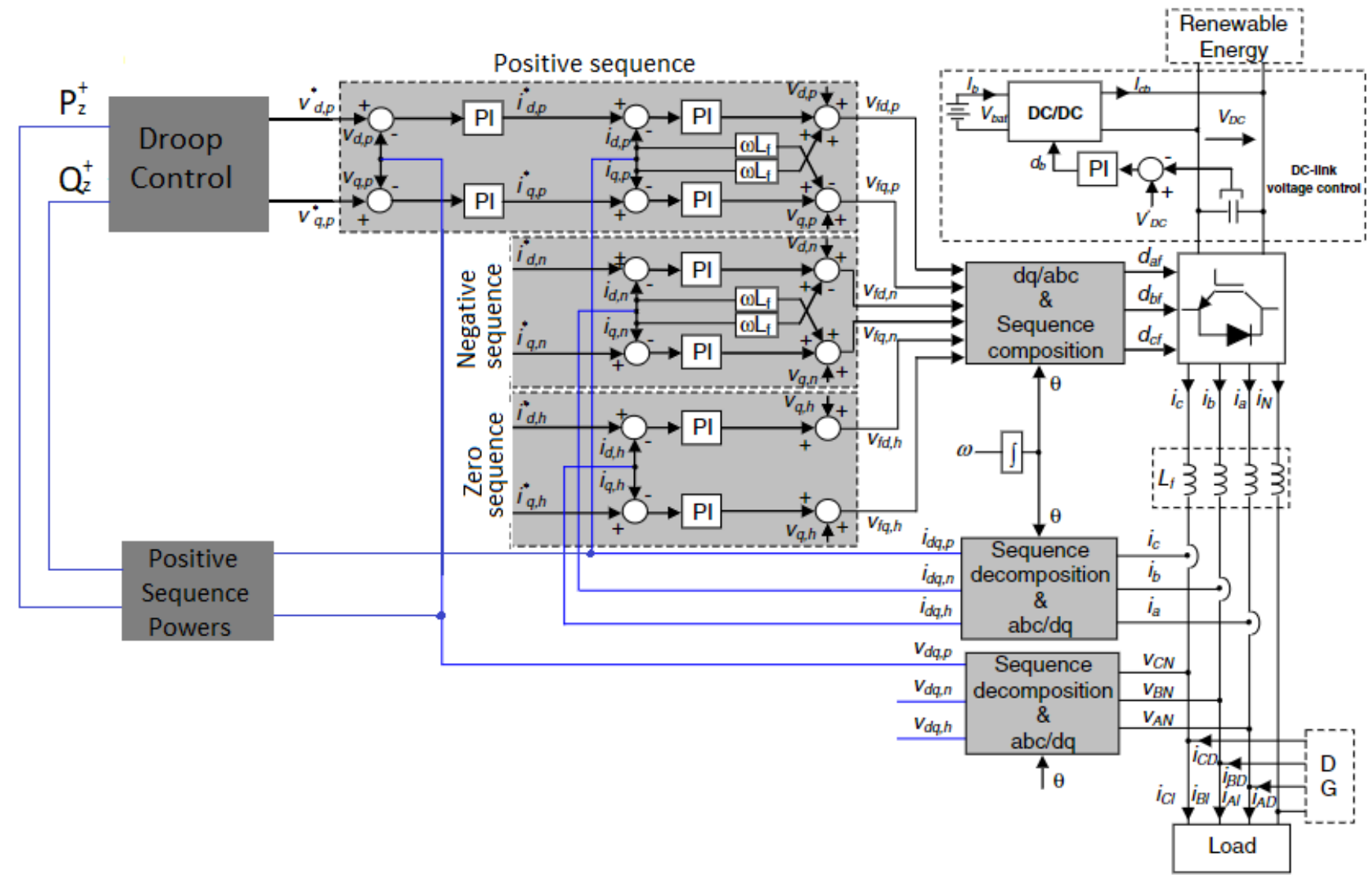

Fig. 3. Schematic diagram of an inverter-based DG in the dq reference frame of different sequence components [19]. 